\title{
A Successful Technique for the Surface Decontamination of Salmonella enterica Serovar Typhimurium Externally Contaminated Whole Shell Eggs Using Common Commercial Kitchen Equipment
}

\author{
Thilini Piushani Keerthirathne, Kirstin Ross, Howard Fallowfield, and Harriet Whiley
}

\begin{abstract}
Eggs are a highly nutritious food source used in a wide range of food products. In Australia, eggs are a frequent source of foodborne salmonellosis outbreaks, associated with eggshell contamination with Salmonella enterica serovar Typhimurium (ST). Despite their potentially hazardous nature, raw eggs are often used and consumed in mayonnaise, mousse, ice cream and eggnog. The aim of this study was to develop a shell egg decontamination method that removed ST contamination from the outside of an egg without impacting its usability. The decontamination method was developed by the adaptation of a temperature-controlled water bath (commonly present in kitchens and associated with the sous-vide technique) for the surface decontamination of eggs. The outside of whole eggs was artificially inoculated with two ST strains. The eggs were decontaminated by placing in a sous-vide cooker with the water heated to $57^{\circ} \mathrm{C}$. The remaining viable ST present on the whole shell egg, crushed shells, internal egg contents and sous-vide water were enumerated over time by culturing onto XLD agar. The quality of the uncontaminated heat-treated eggs was determined by measuring the Haugh unit, yolk index, albumen $\mathrm{pH}$, thermocoagulation, and stability of foam. A blind control study was conducted to assess the acceptability and usability of the treated eggs by chefs and food handlers for the preparation of mayonnaise. Complete decontamination of ST was achieved by treating eggs for $9 \mathrm{~min}$ in the sous-vide cooker $\left(57^{\circ} \mathrm{C}\right)$. No statistically significant difference was observed in the quality of treated eggs compared with nontreated eggs using the quality measurements and acceptability score from chefs. This method provides a simple approach that can be adopted by chefs and food handlers to obtain safe eggs before the preparation of raw egg products.
\end{abstract}

Keywords: salmonellosis, pasteurization, egg quality, mayonnaise, food handler, chef, food safety, sous-vide

\section{Introduction}

$\mathrm{O}_{\mathrm{d}}^{\mathrm{A}}$ ALMONELLOSIS IS A major foodborne gastrointestinal disease that affects public health (Stephen and Barnett, 2017). According to Gibney et al. (2014), in 2010 salmonellosis was among the most common gastrointestinal diseases in Australia, with the main source identified as raw egg products (Gole et al., 2014). In Australia, Salmonella enterica serovar Typhimurium (ST) is the serovar most commonly linked with the egg-related outbreaks (De Buck et al., 2004; Firestone et al., 2007; OzFoodNet, 2009), whereas globally Salmonella enterica serovar Enteritidis (SE) is the main cause (Hendriksen et al., 2011). SE primarily contaminates the internal contents (ICs) of the eggs (Wales and Davies, 2011), whereas ST is found on the outside of the eggshell with internal contamination uncommon (De Buck et al., 2004; Martelli and Davies, 2012).
Previous studies investigating strategies for egg decontamination have focused on eggs internally contaminated with SE (Gast, 1993; Hou et al., 1996; Stadelman et al., 1996; Schuman et al., 1997; Himathongkham et al., 1999). A review conducted by Keerthirathne et al. (2017) identified only one study that investigated an egg decontamination method for ST. However, this study used eggs internally contaminated with ST (Himathongkham et al., 1999) that is not commonly observed.

In addition, the currently available egg pasteurization and decontamination processes can have variable effects on the egg proteins. This influences their coagulation, foaming, and emulsifying properties, ultimately impacting their usability and preventing chefs from using these treated eggs (Keerthirathne et al., 2017).

The aim of this study was to develop a simple decontamination method for eggs externally contaminated with ST that

Environmental Health Group, College of Science and Engineering, Flinders University, Adelaide, Australia. 
does not impact the usability of the eggs. A water bath method using kitchen equipment commonly used for sousvide cooking was selected to ensure easy implementation in a commercial kitchen. A blind control study was also conducted to assess the acceptability and usability of the treated eggs by chefs and food handlers.

\section{Materials and Methods}

Two strains of ST were used for the experiment; a standard strain (STs) of ST (ATCC 53647) and a clinical isolate (STi).

\section{Preparation of the Salmonella culture}

An ST culture was prepared by introducing a typical ST colony from an XLD agar plate (Oxoid Ltd., Basingstoke, United Kingdom) into $100 \mathrm{~mL}$ of peptone water (Oxoid Ltd.). This was incubated for $12 \mathrm{~h}$ at $37^{\circ} \mathrm{C}$ in a shaking incubator (Innova ${ }^{\circledR} 44$, Incubator Shaker Series). After incubation, the broth culture was centrifuged at $1400 \times g$ for $15 \mathrm{~min}$ and the pellet was resuspended in buffered peptone water (BPW; Oxoid Ltd.). The optical density (OD) at $600 \mathrm{~nm}$ (UV-1800, Shimadzu UV-spectrophotometer) was adjusted to 1 using BPW to obtain a concentration of $10^{9}$ colony-forming units (CFU)/mL (Chen et al., 2011) and was serially diluted to $10^{5} \mathrm{CFU} / \mathrm{mL}$.

\section{Inoculation of the shell eggs}

The procedure used was adopted from Gole et al. (2014) with some modifications as stated hereunder. Washed, large, commercial eggs at least 1 month before the use-by date were used for the experiments. The eggs with dirt and cracks were discarded. An egg was immersed in the ST culture $\left(10^{5} \mathrm{CFU} / \mathrm{mL}\right)$ for $90 \mathrm{~s}$ and air dried at room temperature under a safety hood.

\section{Decontamination procedure}

The inoculated eggs were placed directly in the sous-vide apparatus (Duos ${ }^{\mathrm{TM}}$ Sous-Vide; Sunbeam) with water heated to $57^{\circ} \mathrm{C}$ (note: the eggs were not placed in a vacuum-sealed bag as is normal process for sous-vide). The temperature of the water was confirmed using a thermometer at the beginning and the end of the decontamination procedure. Eggs were heat treated for $30 \mathrm{~s}, 1,2,3,3.5,6,6.5,7,8$, and $9 \mathrm{~min}$.

The experiment was conducted with three eggs at each time point. The experiment was repeated three times for each time point and each Salmonella strain, that is, 18 eggs (9 inoculated with STs and 9 with STi) were tested at each time point. Artificially inoculated eggs that did not undergo the heat treatment was used as the control $(\mathrm{T}=0)$. Growth and/or resuscitation was recorded as positive if there was growth in any of the XLD agar plates or broth cultures tested. The results were recorded as negative if there was no growth in any of the plates or broth cultures tested.

\section{Recovery of Salmonella after decontamination}

Recovery of Salmonella after decontamination was enumerated in triplicate for each time point on XLD agar, that is, 9 eggs were processed in triplicate that was equal to 27 spread plates per each time point for each component of the egg (27 plates for intact whole egg, 27 plates for crushed shells, and 27 plates for ICs). The crushed shell and ICs of an egg were processed separately to investigate the potential move- ment of ST throughout the eggshell membrane. All plates and broth media were incubated (Memmert Incubator IN30; Schwabach, Germany) overnight at $37^{\circ} \mathrm{C}$. Colonies with typical ST morphology were counted. The entire experiment was repeated for each Salmonella strain (STs and STi).

Intact whole egg. After the heat treatment, the intact whole egg was placed in a ziplock bag and massaged in $10 \mathrm{~mL}$ BPW for $1 \mathrm{~min}$ to dislodge microorganisms on the external surface of the eggshell (McAuley et al., 2015). One hundred microliters of this solution was spread plated on XLD agar.

Crushed eggshell. The eggs were dipped in freshly prepared $70 \%$ ethanol for $30 \mathrm{~s}$ and dried at room temperature to prevent live bacteria (if any) residing on the eggshell from contaminating the ICs (Gole et al., 2014). This step was conducted to determine whether the bacteria were present in the eggshell membrane or whether it had migrated into the ICs of the egg. The eggs were cracked using a sterile knife. The shells were crushed in $10 \mathrm{~mL} \mathrm{BPW}$ and massaged in a stomacher for 1 min. One hundred microliters of this was plated on XLD agar.

Internal egg contents. The ICs were homogenized until a uniform suspension was obtained. Two milliliters of this solution was added to $8 \mathrm{~mL} \mathrm{BPW}$. One hundred microliters of the IC-BPW mixture was plated on XLD agar using the spread plate method.

Recovery of Salmonella after decontamination using an enrichment step. Recovery of Salmonella after decontamination was also conducted with an enrichment step to induce the recovery of possible viable but nonculturable (VBNC) cells (Reissbrodt et al., 2002) and to increase the limit of detection from 10 to $1 \mathrm{CFU} / \mathrm{mL}$. Enrichment was conducted by adding $1 \mathrm{~mL}$ from each of the whole shell-BPW mixture, crushed shell-BPW mixture, and the IC-BPW mixture to $9 \mathrm{~mL}$ of sterile BPW separately in triplicate (i.e., 3 broths for 9 eggs $=27$ enrichment broth cultures per time point per each component of the egg). Growth in the BPW was determined using a spectrophotometer and confirmed to be ST by plating onto XLD agar.

Salmonella recovery in sous-vide water. The water of the sous-vide was tested to check the viability of any Salmonella cells that may have washed off into the water during the process. One microliters of the sous-vide water was used to inoculate $9 \mathrm{~mL}$ of sterile BPW and this was conducted in triplicate for each time point $(n=9)$.

\section{Egg quality testing}

Uninoculated eggs were used to test the quality parameters. The egg quality was checked after placing the eggs at $57^{\circ} \mathrm{C}$ for $6.5,9,17,20$, and $28 \mathrm{~min}$. The procedures were adapted from Hou et al. (1996) with modifications as described hereunder. The egg quality was also determined for the eggs without any heat treatment, which were used as controls. Foaming ability, foam stability, protein denaturation and albumen $\mathrm{pH}$ (Eutech Instruments pH700), Haugh unit, yolk index, and the difference in weight of the egg before and after the heat treatment were assessed after the heat treatment. Three eggs were used for each time point/control and each test was repeated three times $(n=9)$. 
Each egg was carefully broken onto a leveled glass plate and the height of the egg albumen surrounding the egg yolk and the height of the egg yolk was measured using a micrometer (Mitutoyo). The height of the egg albumen was measured three times in different positions surrounding the egg yolk and the mean value was used for the calculations. The width of the egg yolk was measured using a compass.

Thermocoagulation of the egg albumen after treatment was measured using a spectrophotometer by measuring the $\mathrm{OD}$ at $600 \mathrm{~nm}$ against water.

To measure foam stability and foaming ability, $100 \mathrm{~mL}$ of egg albumen from three eggs from each treatment was used. Egg albumen was homogenized using an electric mixer (Contempo Stick Mixer with beaker) for $3 \mathrm{~min}$ at lowest power. The foam was left to stand for $3 \mathrm{~min}$ and the height of the foam and the drainage volume were measured.

\section{Usability of treated eggs}

Ethical clearance for this part of the experiment was obtained from the Flinders University Social and Behavioural Research Ethics Committee (project No. 7795). Chefs and the food handlers from local restaurants were approached and 10 volunteers were recruited for the study.

Each chef/food handler was provided with three batches (labeled A, B, and C) of three eggs (nine in total) and asked to prepare mayonnaise using their own recipe and each batch separately. The eggs were not inoculated, but two batches had been heat treated for $9 \mathrm{~min}$ at $57^{\circ} \mathrm{C}$ and one batch was untreated. Participants were unaware which batches were treated or untreated.

Once the participants had prepared their mayonnaise, they were asked to assess the overall acceptability, texture, appearance, smell, color, and stability of the mayonnaise using a 9-point hedonic scale where 9 was "like extremely" and 1 was “dislike very much” (Yao et al., 2003).

\section{Statistical analysis}

One-way analysis of variance test was performed on SPSS software (IBM Corp. Released 2017, IBM SPSS Statistics for Windows, version 25.0; IBM Corp., Armonk, NY) to determine whether there was a significant difference in egg quality between the treated and untreated eggs with statistical significance accepted at $p \leq 0.05$. Chi-square tests were performed to understand the heat resistance patterns of the two Salmonella strains used in the experiment. Kruskal-Wallis $\mathrm{H}$ tests were performed on SPSS software to analyze the data obtained from hedonic scales.

\section{Results}

The concentration of Salmonella cells inoculated on to the egg varied from 28 to $150 \mathrm{CFU} / \mathrm{mL}$ with an average of $74 \mathrm{CFU} / \mathrm{mL}$, that is, $7.4 \times 10^{1} \mathrm{CFU} / \mathrm{egg}(n=9)$ that was confirmed following the methods described in the sections Intact whole egg, Crushed eggshell, and Internal egg contents. This inoculation concentration was chosen because previous studies have demonstrated that contaminated eggs typically have between 10 and 100 CFU Salmonella per egg (Humphrey et al., 1989). Previous studies conducted by Hou et al. (1996) and Shenga et al. (2010) on egg decontamination demonstrated that $57^{\circ} \mathrm{C}$ was effective against SE, which is why this temperature was chosen for this study.

\section{Decontamination of the eggs}

There was a significant difference $(p=0.22)$ in resistance to heat between the standard strain (STs) and the clinical isolate (STi). There was no growth of STs in any of the XLD agar plates after $3.5 \mathrm{~min}$ at $57^{\circ} \mathrm{C}$ in the sous-vide water bath but enrichment of the STs confirmed the survival of Salmonella after $3.5 \mathrm{~min}$. No growth of STs was observed after $6 \mathrm{~min}$ at $57^{\circ} \mathrm{C}$ after enrichment (Table 1). STi were not recovered from the eggs after $8 \mathrm{~min}$ in any of the XLD agar plates but the enrichment step confirmed the survival of Salmonella. Nine minutes at $57^{\circ} \mathrm{C}$ confirmed the complete loss of viability of the STi strain in all the XLD agar plates tested (Table 2). Salmonella was not recovered from the sousvide water bath water after the heat treatment for 9 and 6 min for the STi and STs, respectively.

Table 1. Growth of STs After Direct Plating and Enrichment

\begin{tabular}{|c|c|c|c|c|c|c|c|}
\hline \multirow[b]{2}{*}{ Time $(\min )^{a}$} & \multicolumn{3}{|c|}{ XLD plate $S T S$} & \multicolumn{3}{|c|}{ Growth of STs after enrichment in BPW } & \multirow[b]{2}{*}{$\begin{array}{l}\text { Sous-vide } \\
\text { water STs }\end{array}$} \\
\hline & $\begin{array}{c}\text { Whole } \\
\text { shell egg }\end{array}$ & $\begin{array}{c}\text { Crushed } \\
\text { shells }\end{array}$ & $\begin{array}{l}\text { Internal } \\
\text { contents }\end{array}$ & $\begin{array}{c}\text { Whole } \\
\text { shell egg }\end{array}$ & $\begin{array}{c}\text { Crushed } \\
\text { shells }\end{array}$ & $\begin{array}{l}\text { Internal } \\
\text { contents }\end{array}$ & \\
\hline 0 & + & + & - & NA & NA & NA & NA \\
\hline 0.5 & - & - & - & NA & NA & NA & NA \\
\hline 1 & - & - & - & NA & NA & NA & NA \\
\hline 2 & - & - & - & NA & NA & NA & NA \\
\hline 3 & - & - & - & + & + & - & NA \\
\hline 3.5 & - & - & - & + & + & - & NA \\
\hline 6 & - & - & - & - & - & - & - \\
\hline 6.5 & NA & NA & NA & NA & NA & NA & NA \\
\hline 7 & NA & NA & NA & NA & NA & NA & NA \\
\hline 8 & NA & NA & NA & NA & NA & NA & NA \\
\hline 9 & - & - & - & - & - & - & - \\
\hline
\end{tabular}

Total number of eggs processed is $72 .+$, Positive growth on any plate tested; - , no growth in all the plates tested; NA, the points that were not tested.

${ }^{a}$ Nine eggs were used at each time point. The survival of Salmonella was tested in triplicate at each time point (27 spread plates per each time point were tested for each component of the eggs).

BPW, buffered peptone water. 
Table 2. Growth of STi after Direct Plating and Enrichment

\begin{tabular}{|c|c|c|c|c|c|c|c|}
\hline \multirow[b]{2}{*}{ Time $(\min )^{a}$} & \multicolumn{3}{|c|}{$X L D$ plate $S T i$} & \multicolumn{3}{|c|}{ Growth of STi after enrichment in $B P W$} & \multirow[b]{2}{*}{$\begin{array}{l}\text { Sous-vide } \\
\text { water STi }\end{array}$} \\
\hline & $\begin{array}{c}\text { Whole } \\
\text { shell egg }\end{array}$ & $\begin{array}{l}\text { Crushed } \\
\text { shells }\end{array}$ & $\begin{array}{l}\text { Internal } \\
\text { contents }\end{array}$ & $\begin{array}{c}\text { Whole } \\
\text { shell egg }\end{array}$ & $\begin{array}{c}\text { Crushed } \\
\text { shells }\end{array}$ & $\begin{array}{l}\text { Internal } \\
\text { contents }\end{array}$ & \\
\hline 0 & + & + & - & + & + & - & NA \\
\hline 0.5 & + & + & - & + & + & - & - \\
\hline 1 & + & - & - & + & + & - & - \\
\hline 2 & + & + & + & + & + & + & - \\
\hline 3 & + & - & - & + & - & - & - \\
\hline 3.5 & - & - & - & - & + & + & + \\
\hline 6 & + & - & - & + & - & + & - \\
\hline 6.5 & - & - & - & + & - & + & + \\
\hline 7 & + & - & - & + & - & - & - \\
\hline 8 & + & - & - & + & - & - & - \\
\hline 9 & - & - & - & - & - & - & - \\
\hline
\end{tabular}

Total number of eggs processed is $99 .+$, Positive growth on any plate tested; - , no growth in all the plates tested; NA, the points that were not tested.

${ }^{a}$ Nine eggs were used at each time point. The survival of Salmonella was tested in triplicate at each time point (27 spread plates per each time point were tested for each component of the eggs).

BPW, buffered peptone water.

Impact on the egg quality

The average weight difference of the egg before and after the heat treatment was $0.02 \mathrm{~g}$ in all the eggs tested and the difference was not significant $(p=0.92)$ at $9 \mathrm{~min}$ at $57^{\circ} \mathrm{C}$. The $\mathrm{pH}$ of the egg albumen with and without the heat treatment was 9.1 and 9.07 , respectively, after $9 \mathrm{~min}$ at $57^{\circ} \mathrm{C}(p=0.37)$. The average OD reading at $600 \mathrm{~nm}$ for the eggs without the heat treatment was 0.3 , whereas the treated eggs were recorded as 0.8 against water (Fig. 1A), indicating a slight denaturation of the egg albumen but this was not a statistically significant difference $(p=0.17)$ when compared with the control eggs. The average egg yolk index for the control eggs was 0.45 and yolk index of the treated eggs was 0.41 . The average Haugh unit of the control and the treated eggs was 79.41 and 89.45 , respectively. The yolk index and the
A

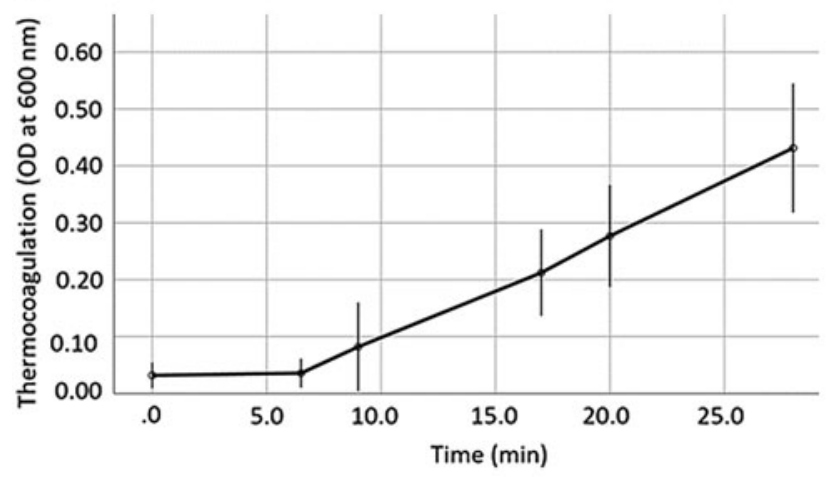

B

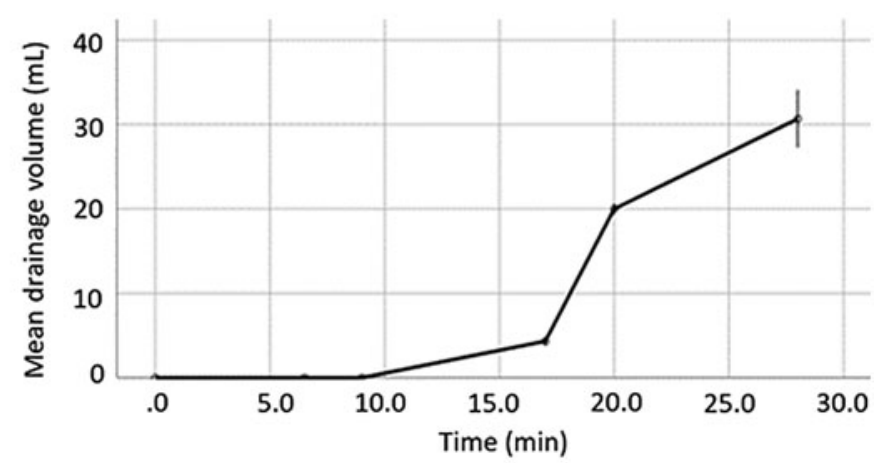

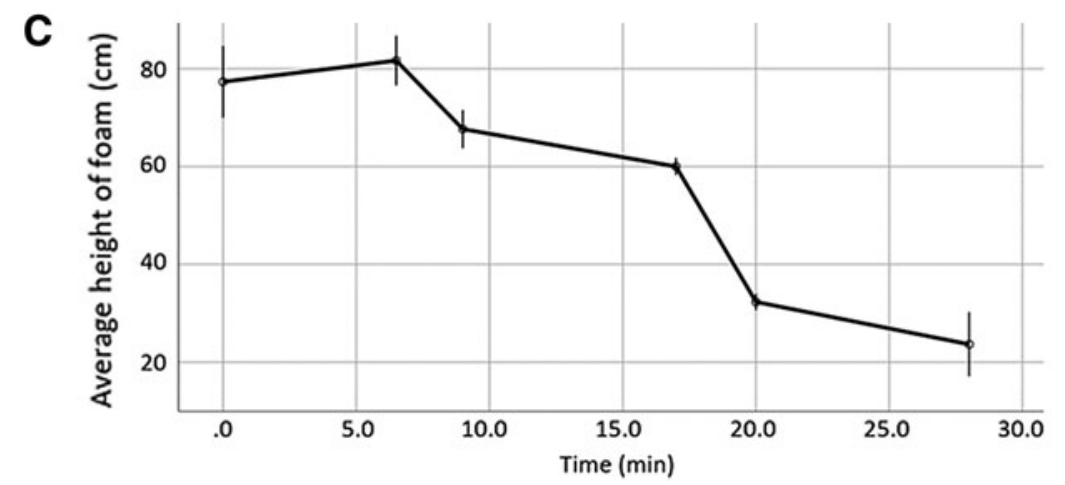

FIG. 1. (A) Thermocoagulation of the egg albumen; (B) change in drainage volume; (C) change in the height of foam produced over time when placed the eggs in water heated to $57^{\circ} \mathrm{C}$. Error bars indicate $95 \%$ confidence interval. 
Haugh unit values were not significantly different from the values of the control eggs $(p=0.16$ and $p=0.053$, respectively). The average height of foam produced by the untreated eggs was $79.4 \mathrm{~cm}$ (Fig. 1B), whereas that of the treated eggs was $67.7 \mathrm{~cm}$, which is not significantly different $(p=0.016)$. There was no drainage volume for the control and the treated eggs (Fig. 1C) at $9 \mathrm{~min}$ at $57^{\circ} \mathrm{C}$.

\section{Usability of the eggs}

Chefs and food handlers rated the mayonnaise made separately from the treated eggs and the untreated eggs. There was no difference observed in the chefs' and food handlers' acceptance of the treated or untreated eggs $(p=0.73)$ (Fig. 2).

Seventy percent $(70 \%)$ and $65 \%$ of the participants rated the overall acceptability and the stability of the mayonnaise made with the treated eggs in the categories "like extremely" and "like very much," respectively. There was no statistical significance between mayonnaise made with the treated and the control eggs when the appearance $(p=0.84)$, texture $(p=0.61)$, smell $(p=0.86)$, color $(p=0.38)$, and the stability ( $p=0.84)$ was considered. Only $5 \%$ of the chefs and food handlers rated the overall acceptability of the prepared mayonnaise as "dislike extremely," whereas $10 \%$ rated the normal eggs in the same category. The results for the stability of the prepared mayonnaise presented a similar pattern.

\section{Discussion}

The developed method inactivated STi after placing inoculated eggs in water heated to $57^{\circ} \mathrm{C}$ for $9 \mathrm{~min}$ without affecting the egg quality. In contrast, complete inactivation of STs was observed after $6 \mathrm{~min}$ at $57^{\circ} \mathrm{C}$. Survival of the STi for a longer time period than the STs indicated that the infectious strain is more resistant to temperature. The enrichment step conducted in this study demonstrated that there was no recovery of VBNC STi after 9 min. This is important as raw eggs could be used in products (such as mousse), which could provide favorable conditions needed for resuscitation and recovery of VBNC cells (Gupte et al., 2003).

Salmonella cells in the VBNC state would not be detected using the culture technique but could subsequently recover and start replicating under favorable environmental conditions (Gupte et al., 2003; Li et al., 2014). The enrichment steps used in this study enabled the detection of any VBNC Salmonella through resuscitation and also increased the detection limit (Reissbrodt et al., 2002).

Previous studies investigating strategies for egg decontamination have focused on eggs internally contaminated with SE (Gast, 1993; Hou et al., 1996; Stadelman et al., 1996; Schuman et al., 1997; Himathongkham et al., 1999). A recent review conducted by Keerthirathne et al. (2017) identified one study that investigated an egg decontamination method for ST. However, this study investigated eggs internally contaminated with ST (Himathongkham et al., 1999), which is not commonly observed. This is the first study to look at decontamination of ST on the eggshell, which is representative of the Australian situation. However, this method may not be as effective for eggs internally contaminated with SE.

To ensure successful implementation of a food safety control mechanism it must be acceptable to chefs and food handlers. Raw eggs are used in many different food products such as mayonnaise, mousse, eggnog, and homemade ice cream. Properties such as gelatinization and emulsification of the eggs are important during the preparation of these food products (Zhao et al., 2010). The stability of the emulsion is a significant property when producing mayonnaise, which is a combination of eggs, vinegar, oil, and spices. Denaturation of the egg proteins because of external means such as heat could have an impact on its functional properties, which

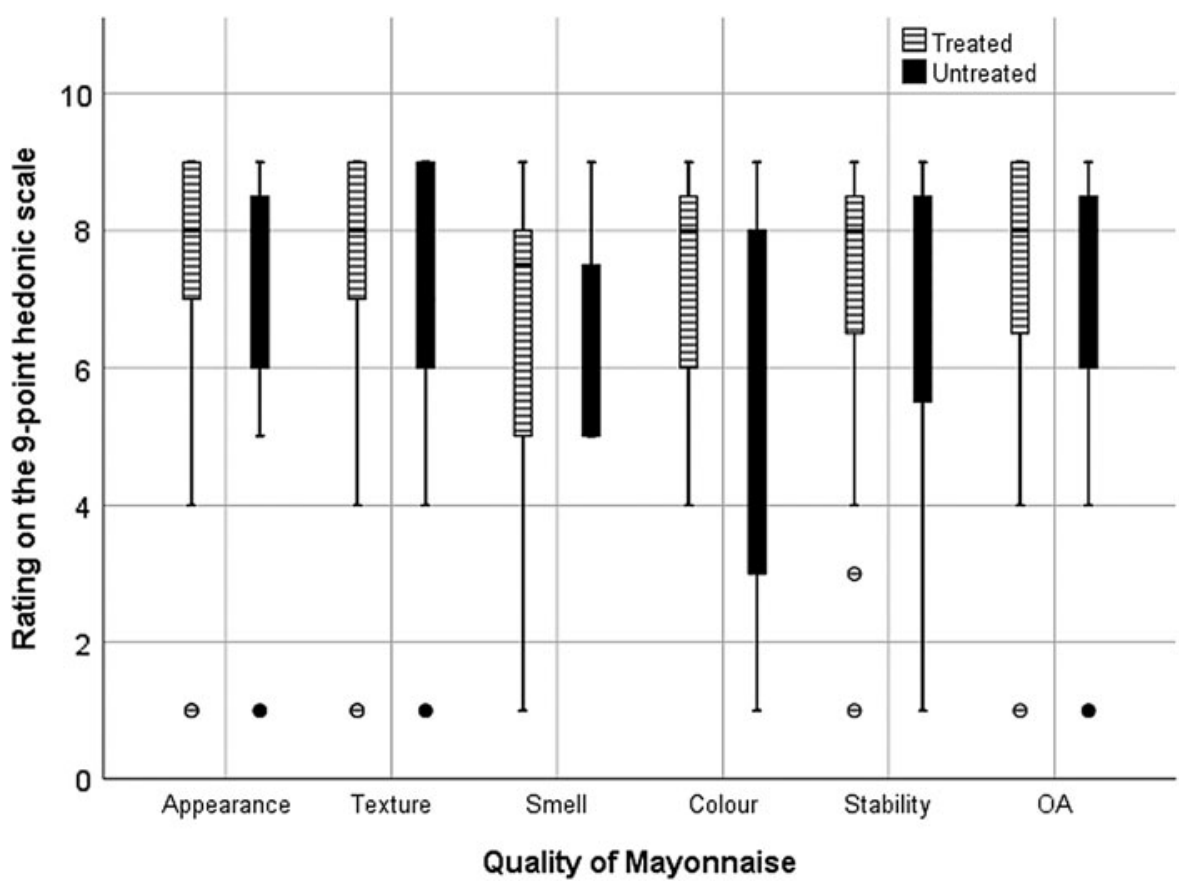

FIG. 2. Clustered boxplot showing the quality according to the 9-point hedonic scale data of the mayonnaise prepared by the chefs and the food handlers using the treated and the untreated eggs. OA, overall acceptability. 
could affect their usability (Campbell et al., 2003). Likewise, Himathongkham et al. (1999) demonstrated cracking of the eggshell after immersion in boiling water for the decontamination of eggs internally contaminated with SE. Another study conducted by Hou et al. (1996) reported that immersion of whole shell eggs in water heated for $57^{\circ} \mathrm{C}$ for 25 to $30 \mathrm{~min}$ denatured the egg albumen.

The egg quality parameters tested indicated that the decontamination method did not impact egg quality. There were no significant differences $(p>0.05)$ between treated and control egg measurements for albumen $\mathrm{pH}$, yolk index, and Haugh unit. These are indicators of the quality of the egg proteins and freshness of the egg and can be affected by temperature (Heath, 1977). Mayonnaise was chosen to examine the chef's acceptability (blind control study) as it has a semisolid consistency, which is stabilized by the egg yolk proteins. As such, the denaturation of the egg proteins could impact the consistency, stability, and the quality of the mayonnaise. There was no difference in the chef's acceptance of the heat treated and untreated eggs, which further supports the findings that this heat-based decontamination method does not affect the physiochemical or functional properties of the eggs.

This decontamination method used kitchen equipment commonly used for sous-vide cooking to ensure easy implementation in a commercial kitchen. This simple and rapid method can be adopted to decontaminate eggs before preparation of raw egg products. Using this method immediately before the preparation of raw egg products could help reduce the burden of salmonellosis in Australia and protect public health. This method could also reduce the possibility of cross-contamination while processing raw egg products.

\section{Future Work}

The method presented in this study provides a simple approach for the decontamination of eggs externally contaminated with ST, which is the most common strain found contaminating Australian eggs. However, future work is needed to evaluate the effectiveness of this method against other Salmonella strains found in Australia. There is also the need for future work exploring the effectiveness of the method against heat resistance-induced strains of Salmonella. This method is designed as a pretreatment to be conducted immediately before the use of an egg. Future research is needed to examine the potential consequences of this method on shelf life and the permeability of the eggshell membrane, which will be of significance if the eggs were not to be used immediately.

\section{Acknowledgments}

The authors acknowledge Helen Hocking of the Salmonella Reference Laboratory, Adelaide, Australia for providing the Salmonella Typhimurium infectious strain and AnnMarie Williams the Quality Assurance Manager of Solar Eggs Pty. Ltd. for cooperating and providing the eggs used for the experiments.

\section{Disclosure Statement}

The authors declare no conflict of interests.

\section{Funding Information}

This research did not receive any specific grant from funding agencies in the public, commercial, or not-for-profit sectors.

\section{References}

Campbell L, Raikos V, Euston SR. Modification of functional properties of egg-white proteins. Nahrung 2003;47: 369-376.

Chen S, Wang F, Beaulieu JC, Stein RE, Ge B. Rapid detection of viable salmonellae in produce by coupling propidium monoazide with loop-mediated isothermal amplification. Appl Environ Microbiol 2011;77:4008-4016.

De Buck J, Van Immerseel F, Haesebrouck F, Ducatelle R. Colonization of the chicken reproductive tract and egg contamination by Salmonella. J Appl Microbiol 2004;97:233-245.

Firestone S, Bell C, Sault C, Stephens N, Lightfoot D. Large outbreaks of Salmonella Typhimurium phage type 135 infections associated with the consumption of products containing raw egg in Tasmania. Commun Dis Intell Q Rep 2007;31:118.

Gast RK. Immersion in boiling water to disinfect egg shells before culturing egg contents for Salmonella Enteritidis. J Food Prot 1993;56:533-535.

Gibney KB, O'Toole J, Sinclair M, Leder K. Disease burden of selected gastrointestinal pathogens in Australia, 2010. Int J Infect Dis 2014;28:176-185.

Gole VC, Chousalkar KK, Roberts JR, Sexton M, May D, Tan J, Kiermeier A. Effect of egg washing and correlation between eggshell characteristics and egg penetration by various Salmonella Typhimurium strains. PLoS One 2014;9:e90987.

Gupte A, De Rezende C, Joseph S. Induction and resuscitation of viable but nonculturable Salmonella enterica serovar Typhimurium DT104. Appl Environ Microbiol 2003;69:66696675.

Heath J. Chemical and related osmotic changes in egg albumen during storage. Poultry Sci 1977;56:822-828.

Hendriksen RS, Vieira AR, Karlsmose S, Lo Fo Wong DM, Jensen AB, Wegener HC, Aarestrup FM. Global monitoring of Salmonella serovar distribution from the World Health Organization Global Foodborne Infections Network Country Data Bank: Results of quality assured laboratories from 2001 to 2007. Foodborne Pathog Dis 2011;8:887-900.

Himathongkham S, Riemann H, Ernst R. Efficacy of disinfection of shell eggs externally contaminated with Salmonella Enteritidis: Implications for egg testing. Int J Food Microbiol 1999;49:161-167.

Hou H, Singh R, Muriana P, Stadelman W. Pasteurization of intact shell eggs. Food Microbiol 1996;13:93-101.

Humphrey T, Baskerville A, Mawer S, Rowe B, Hopper S. Salmonella Enteritidis phage type 4 from the contents of intact eggs: A study involving naturally infected hens. Epidemiol Infect 1989;103:415-423.

Keerthirathne TP, Ross K, Fallowfield H, Whiley H. Reducing risk of salmonellosis through egg decontamination processes. Int J Environ Res Public Health 2017;14:335.

Li L, Mendis N, Trigui H, Oliver JD, Faucher SP. The importance of the viable but non-culturable state in human bacterial pathogens. Front Microbiol 2014;5:258.

Martelli F, Davies RH. Salmonella serovars isolated from table eggs: An overview. Food Res Int 2012;45:745-754.

McAuley CM, Duffy LL, Subasinghe N, Hogg G, Coventry J, Fegan N. Salmonella Typhimurium and Salmonella Sofia: 
Growth in and persistence on eggs under production and retail conditions. Biomed Res Int 2015;2015:914987.

OzFoodNet. Monitoring the incidence and causes of diseases potentially transmitted by food in Australia: Annual report of the OzFoodNet Network, 2008. Commun Dis Intell Q Rep 2009;33:389.

Reissbrodt R, Rienaecker I, Romanova J, Freestone P, Haigh R, Lyte M, Tschäpe H, Williams P. Resuscitation of Salmonella enterica serovar Typhimurium and enterohemorrhagic Escherichia coli from the viable but nonculturable state by heatstable enterobacterial autoinducer. Appl Environ Microbiol 2002;68:4788-4794.

Schuman J, Sheldon B, Vandepopuliere J, Ball H, Jr. Immersion heat treatments for inactivation of Salmonella Enteritidis with intact eggs. J Appl Microbiol 1997;83:438-444.

Shenga E, Singh R, Yadav A. Effect of pasteurization of shell egg on its quality characteristics under ambient storage. J Food Sci Technol 2010;47:420-425.

Stadelman W, Singh R, Muriana P, Hou H. Pasteurization of eggs in the shell. Poultry Sci 1996;75:1122-1125.

Stephen DM, Barnett AG. Using microsimulation to estimate the future health and economic costs of salmonellosis under climate change in Central Queensland, Australia. Environ Health Perspect 2017;125:127001.
Wales A, Davies R. A critical review of Salmonella Typhimurium infection in laying hens. Avian Pathol 2011;40:429436.

Yao E, Lim J, Tamaki K, Ishii R, Kim KO, O’Mahony M. Structured and unstructured 9-point hedonic scales: A cross cultural study with American, Japanese and Korean consumers. J Sens Stud 2003;18:115-139.

Zhao X, Shi-Jian D, Tao G, Xu R, Wang M, Reuhs B, Yang Y. Influence of phospholipase A2 (PLA2)-treated dried egg yolk on wheat dough rheological properties. LWT Food Sci Technol 2010;43:45-51.

Address correspondence to: Thilini Piushani Keerthirathne, MPhil Environmental Health Group College of Science and Engineering Flinders University GPO Box 2100 Adelaide 5001 Australia

E-mail: thilini.ke@flinders.edu.au 